\title{
ESTUDIO PRELIMINAR SOBRE EL USO DE TECNOLOGÍAS DE INFORMACIÓN Y COMUNICACIÓN EN INSTITUCIONES DE EDUCACIÓN SUPERIOR DE LA RED ILUMNO
}

\section{Preliminary study on the use of information and communication technologies in higher education institutions of Ilumno Network}

Luis Alfonso Caro Bautista ${ }^{1}$

Fundación Universitaria del Área Andina, Colombia

Tania Esther Vargas Ordóńez ${ }^{2}$

Jhon Alcides .Campo Urriaga ${ }^{3}$

Recibido: $13-5-2015$

Aceptado: 11-6-2015

\section{Resumen}

El Grupo de Investigación Observatorio Pedagógico de Integración Multimedia OPIM (Colombia) desarrolló el estudio preliminar sobre el uso de Tecnologías de Información y Comunicación TIC, en Educación Virtual en Instituciones de Educación Superior que pertenecen a la red ILUMNO. Partió de la pregunta: ¿Cuáles son las condiciones actuales sobre el uso de Tecnologías de Información y Comunicación TIC en Instituciones de Educación Superior IES de la red ILUMNO? Para responderla, se planteó como objetivo general: Realizar un estudio preliminar sobre el uso de Tecnologías de Información y Comunicación TIC, en Educación Virtual, en las Instituciones de Educación Superior de la red ILUMNO.

El proyecto se realizó en cuatro fases: fase exploratoria, fase de mapeo, fase de muestreo y fase de sistematización y análisis. El grupo de investigación adelantó el estudio centrando su actividad investigativa en cuatro aspectos: (i) pedagógico, relacionado a la existencia y aplicación de modelos pedagógicos en los que se evidenciara el uso de TIC en procesos educativos; (ii) tecnológico, referido a los recursos de software y hardware, la condición de uso de TIC como soporte a los procesos académicos; (iii) comunicativo, relacionado con los sistemas de comunicación soportados por herramientas propias de las TIC en escenarios educativos se destacan elementos como: usabilidad, navegabilidad, amigabilidad y (iv) organizacional, relacionado con la estructura administrativa y organizacional adaptada al contexto del uso de TIC (normatividad, funciones, servicios, extensión). Se analizaron documentos de referencia institucional, que formaron parte del estudio. Se aplicó un instrumento que permitió obtener información por parte de directivos docentes, profesores y los estudiantes adscritos a las IES de la red ILUMNO. Se cruzó la información obtenida para adelantar las conclusiones y recomendaciones que se presentan en este documento

Palabras Clave: Virtualidad, tecnologías de información y comunicación, pedagogía, tecnología.

1Secretario Académico de la Facultad de Educación. Investigador en el Observatorio Pedagógico de Integración Multimedia- OPIM y miembro de la red ILUMNO lcaro@areandina.edu.co

2Maestrante en Ciencias de las Tecnologías y las Telecomunicaciones en la Fundación Universitaria del Área Andina, Colombia

3Maestrante en Educación en la Fundación Universitaria del Área Andina, Colombia. 
Estudio preliminar sobre el uso de tecnologías de información y comunicación en instituciones de educación superior de la Red Ilumno

\section{Abstract}

The research group called Educational Observatory of Multimedia Integration (OPIM) in Colombia, carried out a preliminary study on the use of Information and Communication Technologies ICTs in Virtual Education, in Higher Education Institutions belonging to the ILUMNO network. It started from the question: What are the current conditions on the use of Information and Communication Technologies ICTs in higher education institutions IES belonging to the ILUMNO network? To answer this question, the following general objective was proposed: Conduct a preliminary study on the use of Information and Communication Technologies ICTs, in Virtual Education, in Higher Education Institutions belonging to the ILUMNO network.

The project was carried out in four phases: exploratory phase, mapping phase, sampling phase and the systematization and analysis phase. The research group advanced on the study by focusing their research activities on four areas: (i) educational, related to the existence and implementation of educational models in which the use of ICTs in educational processes is evidenced; (Ii) technology, based on the software and hardware resources, the condition of use of ICTs as support of academic processes; (Iii) communicative, related to the communication systems supported by ICT tools in educational settings where usability, navigability, and user-friendliness are highlighted elements and (iv) organizational, related to the administrative and organizational structure adapted to the context of ICTs use (regulations, functions, services, extension). Institutional reference documents, which formed part of the study, were analyzed. We applied an instrument which made it possible to obtain information from school administrators, teachers and students belonging to the IES of the ILUMNO network. The obtained information was crossed to have the conclusions and recommendations presented in this document in advanced.

Keywords: Virtuality, information and communication technologies, education, technology.

\section{INTRODUCCIÓN}

La incorporación de Tecnologías de Información y comunicación TIC en el sector de la educación ha permitido configurar nuevos escenarios para las prácticas educativas, que son motivo de investigación y estudio en el campo de la pedagogía. Desde los años 90, en América Latina (A.L.) se ha generado una fuerte corriente por parte del sector educativo para incorporar tecnología al interior de las instituciones; recientemente, para estructurar modelos de formación y de educación en lo que se ha llamado la formación en línea y/o educación soportada con TIC. Sin embargo, es relativamente poca la información sobre estudios realizados acerca del uso de las TIC en los sectores educativos y en particular en la educación superior. Por esta razón, el Observatorio Pedagógico de Integración Multimedia (OPIM) planteó el
Estudio preliminar sobre el uso de Tecnologías de Información y Comunicación TIC en la Educación Virtual en Instituciones de Educación Superior que pertenecen a la red ILUMNO, con el fin de generar nueva y mayor información sobre este tema para enriquecer el debate orientado hacia el uso y la apropiación de las TIC en el contexto de la educación superior.

Para ello se consultaron, en primera instancia, documentos generados por instituciones internacionales como el Instituto de Estudios Superiores de América Latina (IESALC), La Organización de las Naciones Unidas para el desarrollo de la Educación la Ciencia y la Cultura (UNESCO), los documentos publicados por la Organización de Estados Iberoamericanos OEI, entre otros.

El grupo de investigadores del OPIM formuló este estudio con el fin de generar información 
acerca de las TIC en educación y, en particular, en lo relacionado con las IES, que conforman la red ILUMNO, a partir de la pregunta: ¿Cuáles son las condiciones actuales sobre el uso de Tecnologías de Información y Comunicación TIC en Instituciones de Educación Superior IES de la red ILUMNO?

\section{Fundamentos teóricos y conceptuales}

Para abordar este estudio, se tomó como referencia elementos teóricos conceptuales desarrollados por Lévy (2010), quien aportó significativamente a la construcción del discurso sobre las tecnologías y sus implicaciones sociales en el contexto de lo virtual. Este autor aportó en lo conceptual y teórico a la construcción de un discurso sobre el significado de lo virtual y las implicaciones que se derivan al incorporar los procesos comunicativos, mediados por las tecnologías, en escenarios educativos, culturales, sociales y económicos. Destaca el hecho de establecer lo que el mismo autor ha llamado, los "colectivos inteligentes", asociados al término de las redes del conocimiento y de la información. Este planteamiento se evidencia en el florecimiento y desarrollo de las redes sociales, que hoy en día han cobrado inusitada importancia, pues se han convertido en verdaderos factores de comunicación permanente entre las personas, rompiendo las barreras de la comunicación tradicional y estableciendo nuevos códigos, nuevos elementos semióticos y semiológicos propios de aspectos comunicacionales de corte superior.

De otro lado, Bateson (1984) desarrolló unos principios epistemológicos sobre la tecnología y las implicaciones en educación. Describe los diferentes estadios en los cuales la tecnología y su desarrollo se convierten en referentes que vinculados de manera directa a las revoluciones científicas. El autor establece elementos para el análisis y la conceptualización sobre el uso de las tecnologías, desde la perspectiva misma de su evolución y desarrollo, pero asociadas de manera directa a lo comunicacional; señala que la comunicación es el eje sobre el cual las personas conforman redes que "fluyen" de manera holística y en las cuales encajan todas las actividades humanas.

Asimismo, Deleuze y Guattari (1990) aproximan los conceptos de rizoma y desterritorialización a los sistemas cibernéticos e informáticos y avanzan en los aportes discursivos y epistemológicos relacionados con las tecnologías y sus impactos en las sociedades. También establecen el criterio del uso de las tecnologías desde la perspectiva de lo socio-ético y comportamental, muy aplicado a los fenómenos sociales de comunicación en el entorno social y las consideraciones culturales que de ello se derivan.

McLuhan (1967) aborda las técnicas de comunicación y las consideraciones propias de los medios y de la prolongación de los sentidos en lo referente a lo virtual. Serres (1994) hace aportes relacionados con la epistemología de las ciencias y la consideración de las TIC como elementos complificadores de los procesos de comunicación social, los aportes investigativos y las reconsideraciones del sujeto y el objeto del conocimiento. Por su parte, Virilio (2011) plantea la condición de la virtualidad como un estado propio de la tendencia hacia la miniaturización del mundo y restableció el concepto de la aceleración, para aplicarlo al contexto de la rapidez en las comunicaciones, generándose una nueva visión sobre el comportamiento del individuo en sociedades posmodernas

Aguilar (2009), en el texto Ontología Cyborg, presenta una nueva teoría con respecto al concepto de actitudes y emociones generados por la virtualidad, al crear una serie de relaciones interpersonales que impactan de manera directa a las comunicaciones entre las personas. Aguilar formula que las redes sociales y sus sistemas comunicacionales serán orientadoras de nuevas dinámicas en el mundo contemporáneo.

De otra parte, se han publicado recientemente documentos relacionados con el estado del arte de la educación y su relación con el uso de TIC en América Latina, uno de ellos, titulado La Educación a Distancia y virtual en Ecuador (Rama, 2013), en donde se resaltan los avances que ha tenido el país en el desarrollo de propuestas de educación soportadas por tecnologías, el uso de TIC y el impacto que estas modalidades de educación han tenido en lo relacionado con la cobertura y la calidad educativa. 
Estudio preliminar sobre el uso de tecnologías de información y comunicación en instituciones de educación superior de la Red Ilumno

Se abordó el documento firmado por cerca de 1300 rectores de 33 países reunidos en el III Encuentro Internacional de Universia 2014 (RIO 2014), donde se destaca el compromiso de las universidades por la generación de nuevos espacios para la consolidación de los modelos de educación virtual y el uso de TIC en el campo de la educación. En el desarrollo de este estudio, y en particular en la fase de diagnóstico, se encontraron varios documentos, sobre los cuales en cierta medida se han visto influenciadas las Instituciones de Educación Superior (IES), entre ellas las congregadas bajo la red ILUMNO. Se reseñan los documentos estudiados: Aprender y enseñar en la cultura digital, en el marco del VII Foro Latinoamericano de Educación Experiencias y aplicaciones en el aula, aprender y enseñar con nuevas tecnologías (2010). En este foro, la autora del documento básico, Inés Dussel (2010), presenta en primera instancia un diagnóstico sobre la expansión de las tecnologías en el contexto escolar y resalta cómo en países como Argentina y Chile se presenta una experiencia relacionada al modelo 1:1 (una computadora por alumno). Igualmente hace referencia a la dotación de elementos electrónicos en los salones de clase: "pizarras electrónicas, los laboratorios de informática móviles, o incluso la convivencia cotidiana con celulares y otros artefactos digitales, muestran que las nuevas tecnologías llegaron para quedarse" (p. 11). Esta afirmación representa en gran medida, el esfuerzo de las instituciones gubernamentales de los países de A.L. de dotar de equipos a las instituciones educativas para luego pensar que hacer con ellos.

Un segundo aspecto es el relacionado con la formación de los docentes y la creación de nuevos escenarios para sus prácticas, orientadas de una parte a la transformación de currículos, a la pertinencia de los contenidos y otro, a la presentación de las herramientas que pueden facilitar y soportar los procesos de formación. Al respecto la autora afirma que "no es suficiente con dotar a las escuelas con computadoras o con acceso a Internet: también es necesario trabajar en la formación docente y en la formulación de nuevos repertorios de prácticas que permitan hacer usos más complejos y significativos de los medios digitales" (p. 12).
En tercer lugar, la autora destaca lo relacionado con el salto tecnológico, haciendo referencia a la permanente evolución de las TIC como una generación de tecnologías que son frecuentemente cambiadas (p. 18) por otra con mejores condiciones de usabilidad.

De otra parte el Fondo de las Naciones Unidas para la Infancia (UNICEF) presenta el Informe sistemas de formación docente inicial y continua para la Educación Básica en América Latina (2013). En este documento se hace un balance sobre la incorporación de TIC en el contexto escolar y se relacionas aspectos de vital importancia como es la formación docente para fortalecer el uso y la apropiación de estas tecnologías, en su desempeño profesional.

Con respecto a lo relacionado con la educación superior, la Organización para la Cooperación y el Desarrollo Económicos (OCDE), conjuntamente con el Banco Mundial presentan un informe sobre los estados presente y futuro de la educación superior en Colombia (2012). En dicho informe se reconocen las fortalezas y debilidades del sistema de educación del país y las asocian con los resultados de las evaluaciones internas y externas con relación a las competencias de los profesionales y los estándares internacionales. Se hace un llamado para que los sistemas educativos del país se transformen y se orienten hacia la modernización de los currículos, su flexibilidad, la internacionalización, y en particular al uso intensivo de las tecnologías para consolidar las metas de permanencia y cobertura de la educación superior en América Latina.

Desde esta perspectiva se planteó la pregunta: ¿Cuáles son las condiciones actuales sobre el uso de Tecnologías de Información y Comunicación TIC en Instituciones de Educación Superior IES de la red ILUMNO? El objetivo general fue realizar un estudio preliminar sobre el uso de Tecnologías de Información y Comunicación (TIC) en Instituciones de Educación Superior que pertenecen a la red ILUMNO. Entre los objetivos específicos se consideraron: (i) identificar los factores pedagógicos que rodean el uso de Tecnologías de Información y Comunicación en Instituciones de Educación Superior que pertenecen a la red 
ILUMNO, (ii) establecer las características tecnológicas sobre las cuales se genera el uso de las Tecnologías de Información y Comunicación en Instituciones de Educación Superior que pertenecen a la red ILUMNO y (iii) reconocer las condiciones sobre las cuales se establecen las relaciones comunicativas y organizacionales, que permiten el uso de las Tecnologías de Información y Comunicación, en Instituciones de Educación Superior que pertenecen a la red ILUMNO.

\section{MATERIALES Y MÉTODo}

\section{Población de estudio}

La población de estudio estuvo constituida por académicos, vicerrectores, directores, coordinadores y estudiantes de las nueve IES que conforman la red ILUMNO del nivel de pregrado y posgrado. Se trabajó con una muestra no probabilística por conveniencia conformada por 9 vicerrectores académicos, 4 directores de programas (dos de pregrado y dos posgrado, por institución), 6 coordinadores de programa (tres por cada programa: pregrado y posgrado), 9 directores de proyectos virtuales (uno por cada institución), una muestra significativa de profesores vinculados a procesos virtuales (diez por institución) y una muestra significativa de estudiantes virtuales (cinco a diez por institución).

Los actores pueden haber cambiado en cada entidad de acuerdo a las condiciones y características propias de las Instituciones.

\section{Instrumentos}

Los instrumentos fueron diseñados por los investigadores del grupo Observatorio Pedagógico de Integración Multimedia (OPIM) y se ajustan a las necesidades del proyecto y las condiciones propias de cada institución. Estos se validaron con la opinión de profesionales expertos en el diseño de instrumentos y el análisis cualitativo y cuantitativo.

La información recibida se analizó desde la pers- pectiva transdisciplinar y desde la reflexión compleja de uno o varios actores sociales, "sobre las prácticas para aprender de ellas" con base en la contrastación y la realidad del contexto educativo local, desde el enfoque histórico-crítico, interpretado y sistematizado para visualizar los diferentes escenarios tradicionales, emergentes y/o alternativos y contrastarlos con aquellos sobre los cuales se tiene mayor dominio informativo y comunicativo.

Tabla 1. Operacionalización de la variable usos y apropiación de tic en procesos educativos

\begin{tabular}{lll}
\multicolumn{1}{c}{ Variable } & \multicolumn{1}{c}{ Factores } & \multicolumn{1}{c}{ Indicadores } \\
$\begin{array}{l}\text { Usos y apro- } \\
\text { piación de TIC } \\
\text { en procesos } \\
\text { educativos }\end{array}$ & Pedagógicos & $\begin{array}{l}\text { Didáctica y } \\
\text { Metodología } \\
\text { Equipos software } \\
\text { y hardware }\end{array}$ \\
& $\begin{array}{l}\text { Organizacio- } \\
\text { nales }\end{array}$ & $\begin{array}{l}\text { Estructuras } \\
\text { administrativas y } \\
\text { académicas }\end{array}$ \\
& Comunicativo & $\begin{array}{l}\text { Imagen institu- } \\
\text { cional }\end{array}$ \\
& Usabilidad & $\begin{array}{l}\text { Acceso } \\
\text { Pertinencia }\end{array}$ \\
Aplicabilidad & $\begin{array}{l}\text { Navegabilidad } \\
\text { Amigabilidad } \\
\text { Diseño }\end{array}$ & $\begin{array}{l}\text { Navegación } \\
\text { Información } \\
\text { pertinencia }\end{array}$ \\
& Funcionabilidad &
\end{tabular}

\section{Tipo y diseño}

Por tratarse de un estudio sobre estados del arte aplicados al contexto de la educación virtual, se aplica una metodología con enfoque mixto (cualitativo-cuantitativo), que permite realizar un estudio comparativo a partir de la información obtenida con base en la lectura de los portales de cada una de las nueve IES que conforman la red ILUMNO y cruzar la información con la obtenida, usando métodos estadísticos.

\section{Procedimiento}

Para la realización de este proyecto, se establecieron cuatro fases que posibilitaron la obtención de la información que se detalla a continuación:

Fase 1: Investigación documental (fase exploratoria). Se tomó como referente la información de 
Estudio preliminar sobre el uso de tecnologías de información y comunicación en instituciones de educación superior de la Red Ilumno

carácter público que aparece en los sitios web (portales) de cada institución, bajo los siguientes parámetros:

(i) Infraestructura informática. Hace referencia a la capacidad tecnológica, recursos, dispositivos, conectividad, plataformas, que posibilitan abordar un estudio sobre el uso de tic en esta muestra de IES.

(ii) Caracterización de los programas académicos. Se hizo énfasis en el análisis de información relacionada con las áreas pedagógicas y curriculares, orientando el estudio hacia el uso de TIC en procesos educativos.

Fase 2: Investigación de campo (fase de mapeo - muestreo). Una vez diseñados por los investigadores del grupo Observatorio Pedagógico de Integración Multimedia (OPIM) y avalados por especialistas externos, los instrumentos para recolección de información a los estudiantes, docentes y directivos docentes se aplicaron en cada institución observada.

Fase 3: Sistematización análisis e interpretación de resultados. Se abordó desde un enfoque interpretativo y crítico, que consistió en el análisis y la interpretación de la información. Esta metodología permitió que los investigadores circulen sobre uno o varios horizontes conceptuales que contribuyan de manera holística a la construcción colectiva de ideas y conceptos propios de las problemáticas estudiadas, a partir de las descripciones de estructuras y contenidos, con el fin de comprender y valorar las realidades propias de cada institución.

Fase 4: Conclusiones y recomendaciones. En esta fase, el grupo de investigadores procedió a realizar las síntesis integradoras del estudio. Esta síntesis se produjo a partir de las confrontaciones conceptuales, los imaginarios e ideas previas de los investigadores y su grupo frente a las realidades y los resultados obtenidos. Luego de la aplicación de los instrumentos diseñados para la recolección de la información, los documentos producto del estudio se ordenaron utilizando los formatos establecidos, para facilitar su difusión y publicación; asimismo, se establecieron las recomendaciones que los investigadores consideraron pertinentes, para que sean tomadas como referentes teóricos, conceptuales, analíticos, y procedimentales orien- tados al fortalecimiento de los procesos de innovación de las IES observadas, y que corresponden al uso de TIC en cada una de ellas. Por tratarse de un estudio sobre el uso de TIC en Instituciones de Educación Superior (IES), se aplicó una metodología con enfoque cualitativo que permitió realizar un estudio a partir de la información obtenida con base en la lectura de los portales de las IES que conforman la red ILUMNO; todo ello complementado con la aplicación de una encuesta orientada a la búsqueda de información que permitiera establecer evidencias sobre el uso de TIC en cada una de ellas. Como muestra representativa se determinó consultar varios de los estamentos que de manera directa pueden ofrecer información correspondiente a la pregunta de investigación. Esta muestra comprendió: coordinadores de programa directores de proyectos virtuales, profesores, estudiantes.

Los actores encuestados pudieron cambiar en cada institución de acuerdo a las condiciones y sus características propias. El estudio se desarrolló en las fases anteriormente expuestas: (i) fase exploratoria, orientada hacia la documentación de la realidad objeto de estudio, para este caso se aplicó el criterio de la información publicada en las páginas y portales web de cada institución; (ii) fase de mapeo, orientada hacia la revisión documental de la información pública enfatizando en el uso de TIC en cada una de las IES observadas; (iii) fase de muestreo, en la que se incluyó a las personas que desempeñan ciertas funciones a nivel institucional y sobre las cuales se puede considerar puntos de vista y nuevas perspectivas en torno al problema planteado; finalmente la fase de sistematización y análisis, que permitió la recopilación, ordenamiento, análisis y síntesis de información, para aproximar conclusiones y recomendaciones.

\section{Resultados}

En el marco del desarrollo del estudio preliminar sobre el uso de Tecnologías de Información y Comunicación TIC en Instituciones de Educación Superior que pertenecen a la red ILUMNO, se tomó como referencia la indagación documental 
y la revisión de cada uno de los portales institucionales, en los cuales se encontraron elementos útiles para abordar el estudio sobre la conceptualización acerca del uso de tecnologías. Esta revisión se orientó desde cuatro ejes: pedagógico, comunicativo, organizacional y tecnológico. En estos se destacan los siguientes factores:

Factor comunicativo. Al ingresar a los web site de cada una de las IES, se observaron los siguientes aspectos:

El portal se divide en tres partes:

- Una que contiene la información relacionada con la universidad, visión y misión; características de los programas académicos que ofrecen y los beneficios educativos y de otra índole que ofrecen las instituciones y que están relacionadas con el bienestar institucional, tales como: emergencias médicas, psicología, gimnasio, actividades recreativas y deportes.

- Otra relacionada con la información referente a los trámites administrativos, tales como: inscripción, procesos de matrícula entre otros.

- Una tercera relacionada con la plataforma educativa, a la cual ingresan estudiantes y docentes con el uso de su clave personal. Por otro lado, se pudo observar que todas las universidades estudiadas hacen uso de las redes sociales como estrategia para darse a conocer, siendo los medios más importantes: Facebook, Twitter, Google, Linkedin entre otros.

Otras estrategias de comunicación que cobran gran importancia y que aparecen en la página web institucional son:

- Medios de información digitales: UDI News (Universidad del Istmo - Panamá),

- Revista Virtual Identidad 21 (Universidad Siglo XXI Argentina). Zona R Publiandina (Área Andina. Colombia),

- Radio virtual Área Andina (Colombia).

Fuerte presencia del portal YouTube como estrategia de comunicación y promoción para dar a conocer las novedades en cuanto a programas académicos se refiere. Para fomentar el aprendizaje de inglés, las universidades utilizan el módulo de Rosetta Stone.
Factor organizacional. En cuanto al aspecto organizativo, todos los sitios web hacen una semblanza institucional, misión y visión de la universidad. También muestran el componente legal de los programas y los vinculan a las políticas leyes y normas de orden nacional. Asimismo, hacen públicos los documentos reglamentarios de orden institucional que existen en cada una de las IES.

En lo relacionado con matrículas, las plataformas permiten el ingreso de datos de la persona interesada para obtener información sobre los programas académicos. Esto permite también acceder a los datos del interesado para desarrollar procesos de promoción, bien por vía telefónica o bien por correo. Algunas universidades cuentan con el proceso de matrícula en línea, otras permiten que el aspirante acceda al formulario para llevarlo a la universidad. Todos los sitios web consultados permiten que el aspirante conozca las ayudas financieras con que cuenta la universidad (préstamos bancarios, convenios, ayudas financieras, descuentos por pronto pago, etcétera).

Se realizó una caracterización de los programas académicos soportados por plataforma: cada IES tiene una oferta académica en diferentes modalidades, entre las que podemos mencionar:

UDI de Panamá, que maneja la modalidad denominada Global Learning, que cuenta a su vez con tres tipos de programas ${ }^{4}$ :

- El programa presencial en el cual el estudiante asiste una vez a la semana a la universidad, donde toma una tele-clase, con profesores de primer nivel, líderes en el sector empresarial y educativo;

- El programa a distancia como sistema de estudios que permite desarrollar sus conocimientos desde el lugar y horario que le resulte más conveniente; y

- El programa virtual cuyo modelo se apoya en el uso de tecnología a través de un espacio virtual.

Siglo XXI de Argentina, que ofrece 5 modalidades en educación, las dos primeras son presenciales y la sénior, que no están soportadas significativamente por tecnologías, como sí lo están las tres

4 http://www.udi.edu/como-funciona-udi-global-learning 
Estudio preliminar sobre el uso de tecnologías de información y comunicación en instituciones de educación superior de la Red Ilumno

siguientes ${ }^{5}$ :

- Educación distribuida. Cursada por Internet, a distancia, con tele-clases en aula, en Centros de Aprendizaje de todo el país.

- Educación Distribuida Home. Cursado por Internet desde cualquier punto del país, contacto online permanente con profesores y trabajo colaborativo con compañeros.

- Virtual posgrados. Varía de acuerdo a cada programa. Puede ser $100 \%$ online o combinar los cursos online con clases quincenales, mensuales o intensivas.

Unijorge de Brasil. Se caracteriza por tener programas virtuales de pregrado y posgrado con reuniones virtuales entre estudiantes y docentes. Esta modalidad se denomina educación a distancia.

USAM de Costa Rica. Maneja programas 90\% virtual y $10 \%$ presencial. Tiene algunos diplomados $100 \%$ virtuales.

La IPP de Chile cuenta con una modalidad semipresencial, que utiliza un campus virtual con toda la información pertinente para el alumno como: horarios, facilidades financieras, un promedio de seis sesiones presenciales por bimestre para desarrollar talleres, exámenes y fomentar la interacción entre los estudiantes y una plataforma por la cual los estudiantes pueden acceder a todos los contenidos multimediales.

Universidad Americana de Paraguay. "La Universidad ofrece Carreras de Grado en el Sistema de Educación Virtual, mediante dos modalidades: A Distancia, Presencial y Semi Presencial a Distancia, adoptando un régimen bimestral, incluyendo las evaluaciones parciales y finales, presenciales o a distancia, según la modalidad inscrita por el estudiante". La modalidad de Educación Virtual "a distancia" se caracteriza por la estimulación de un rol activo del estudiante ante el aprendizaje, la interacción permanente con el docente y el tutor virtual, así como con otros estudiantes, a través de actividades programadas en internet, accediendo a bibliotecas digitales, mediando la Plataforma Educativa Virtual y contando con el apoyo de clases en formato audiovisual y materiales de auto aprendizaje. ${ }^{\prime \prime}$

5 http://www.21.edu.ar/modalidades.html

$6 \mathrm{http}: / /$ www.uamericana.edu.py/modalidades/semi-presencial-a-dis-
Las Instituciones: Fundación Universitaria del Área Andina y Politécnico Grancolombiano manejan algunos programas $100 \%$ virtuales soportados por plataforma EPIC y combinada con plataforma Moodle. Para el caso de los programas virtuales se cuenta con una plataforma (EPIC) que está configurada desde la perspectiva de la producción de contenidos educativos.

Se abordó la lectura y análisis de los resultados de la encuesta aplicada a los actores educativos de las IES de la red ILUMNO, encontrándose información relevante que permitió considerar aspectos muy importantes. Los directivos consideran que tienen una gran experiencia en el uso de las TIC en el desarrollo de actividades administrativas. En general se observa que los directivos de las universidades fuera de Colombia consideran que tienen mayor experiencia en el uso de TIC. Ellos también consideran que a partir del uso de las mismas han podido capacitarse adecuadamente. En lo que respecta a la integración de las TIC en las actividades diarias de orden organizacional, los directivos consideran o perciben que estas son de ayuda en sus actividades diarias, y las vuelve más motivadoras y productivas. Por otro lado, en lo referido a las comunicaciones al interior de cada una de las instituciones, es alta la percepción de estos directivos frente a la eficiencia en la mejora de la comunicación al interior de las instituciones y a la posibilidad de evaluar al personal por medio de herramientas TIC. En cuanto al conocimiento de los lineamientos básicos para conocer el uso de las TIC en la institución, la percepción de conocimiento de las mismas es regular al igual que lo que sucede con los recursos de software administrativo, la percepción de tenerlos es buena pero no totalmente. En la figura 1 se muestra que los directivos consideran que tienen una gran experiencia en el uso de las TIC en el desarrollo de actividades administrativas. En general se observa que los directivos de las universidades fuera de Colombia consideran que tienen mayor experiencia en el uso de TIC y que a partir del uso de las mismas han podido capacitarse adecuadamente.

Respecto a la integración de las TIC en las actividades diarias de orden organizacional, los directivos consideran o perciben que estas son

tancia/ 

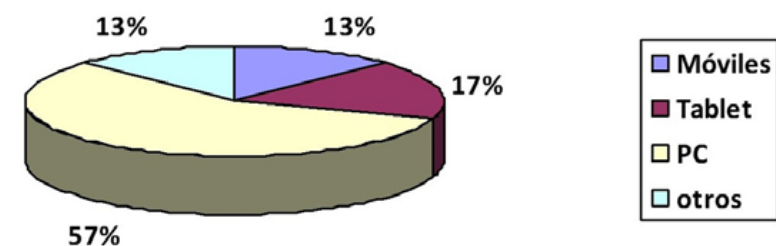

Figura 1. Elementos con los que cuenta el personal administrativo para acceder al uso de TIC

de ayuda en sus actividades diarias, y las vuelve más motivadoras y productivas. Como se puede observar en la figura 2. De otra parte, respecto a las comunicaciones al interior de cada una de las instituciones, es alta la percepción de estos directivos frente a la eficiencia en la mejora de la comunicación al interior de las instituciones y a la posibilidad de evaluar al personal por medio de herramientas TIC. En cuanto al conocimiento de los lineamientos básicos para conocer el uso de las TIC en la institución, la percepción de conocimiento de las mismas es regular, al igual que lo que sucede con los recursos de software administrativo, la percepción de tenerlos es buena, pero no totalmente.
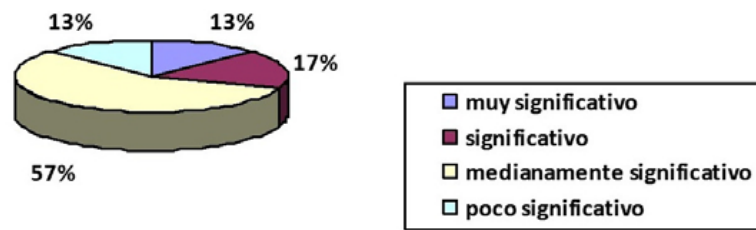

Figura 2. Percepción sobre el uso de TIC en actividades propias de la gestión administrativa

Factor tecnológico. Se desarrolló teniendo en cuenta los resultados de las encuestas realizadas a algunos directivos, docentes y estudiantes de las instituciones que conforman la Red ILUMNO (ver batería de preguntas apéndice A).

Las respuestas de la encuesta según el aspecto técnico se presentan en una matriz de resultados con su respectivo análisis.

Análisis de resultados encuesta directivos y docentes sobre uso de TIC en su entorno educativo.

La Matriz de Resultados Directivos. Las preguntas del directivo están divididas en 3 tres grupos se- gún la batería de preguntas (figura 3). El análisis realizado permitió percibir que los directivos utilizan equipos y recursos tecnológicos (computadores, portátiles, dispositivos móviles e internet) en un grado muy alto. Utilizan las TIC como herramientas para evaluar a las personas a cargo en un nivel intermedio. Los directivos ven en un alto grado de importancia las necesidades de formación para integrar las TIC en su rol que desempeña en la institución para conocer mejor el entorno web de la Institución. En cuanto a conocer sitios web específicos para integrarlos en la docencia de aula, los directivos lo perciben de una forma alta. Respecto a aprender a utilizar las TIC en aula con entornos de aprendizaje virtual, cursos semipresenciales, entre otros, los directivos ven en un alto grado de importancia la oportunidad de conocer propuestas didácticas de integración de TIC. Con respecto a la vigilancia tecnológica, inteligencia de mercados, inteligencia competitiva se percibe en un nivel intermedio de importancia.
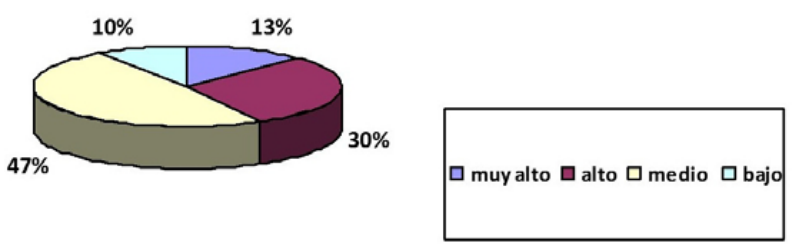

Figura 3. Utilización de TIC en aspectos académicos-informáticos

Matriz de Resultados Docentes. Las preguntas del docente están divididas en siete (7) grupos según la batería de preguntas.

El análisis de los resultados mostrados en las figuras 4, 5, 6, 7 y 8) deja en evidencia que los docentes cuentan con equipos tecnológicos y móviles en un grado alto para sus labores diarias y en el aula de clase. Con respecto al uso de tic, los docentes muestran altos niveles de formación y aplicabilidad a los procesos de aprendizaje-enseñanza. La integración de las TIC en el aula es un medio para hacer clases más motivadoras para estudiantes y docentes se perciben un nivel alto. En un análisis general con respecto al uso de las TIC para evaluar a los estudiantes, los docentes consideran este factor en un nivel alto.

Con respecto a las estrategias pedagógicas, se debe 
Estudio preliminar sobre el uso de tecnologías de información y comunicación en instituciones de educación superior de la Red Ilumno

fortalecer trabajos colaborativos o en grupos, con apoyo de computador (redes, wikis etcétera) y autoaprendizaje a través de la enseñanza asistida por computador. Además los docentes deben fortalecer el uso de aulas virtuales, cuyo uso es de nivel medio.
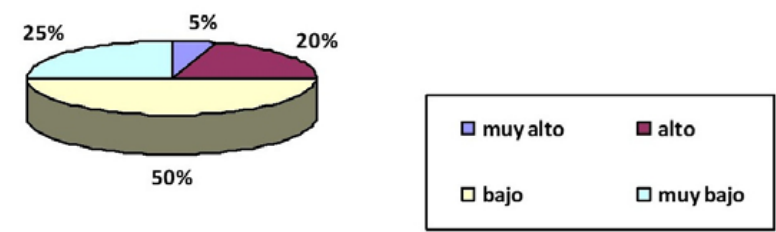

Figura 4. Componente técnico uso de TIC en actividades académicas
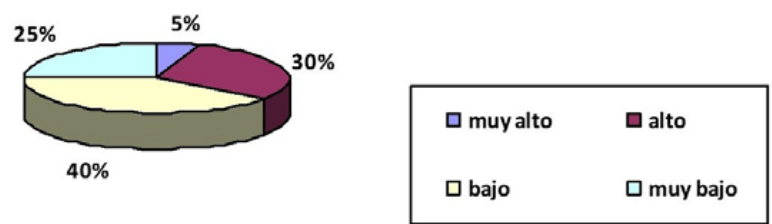

Figura 5. Componente técnico uso de TIC en labores docentes

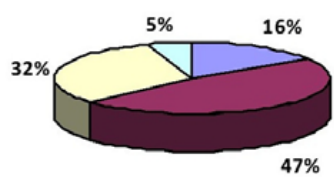

\begin{tabular}{|ll|}
\hline$\square$ muy alto & $\square$ alto \\
$\square$ bajo & $\square$ muy bajo \\
\hline
\end{tabular}

Figura 6. Componente pedagógico, estrategias y didácticas con uso de TIC
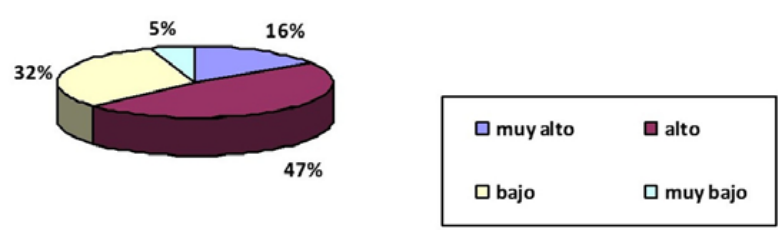

Figura 7. Componentes organizacional. Organización de actividades docentes con uso de TIC
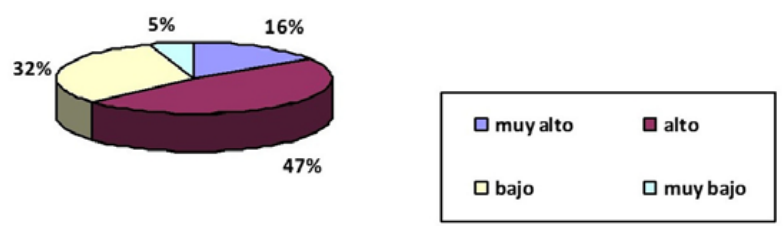

Figura 8. Componente administrativo. Actividades docentes con uso de TIC
En lo relacionado con los recursos (video beam, software educativo, páginas web, portales, comunidades, laboratorios virtuales correo electrónico, televisor, vídeo, DVD) para el apoyo en el aula de clase hay una buena apreciación. De igual manera, la utilización de plataformas y entornos de aprendizaje (Moodle, Educampus, Atutor, Epic, otros) se evidencia un nivel alto de utilización de estos recursos.

En cuanto al manejo de editores de audio, vídeo, animación, imágenes, hipertextos, etcétera, se debe fortalecer para que el nivel llegue al más alto y lo puedan aplicar en el aula de clase. El desarrollo de materiales electrónicos de elaboración propia (como web, software, guías, etcétera) se encuentra en un nivel bueno, ya que en cada universidad se viene animando a los estudiantes en la producción propia.

Con respecto a la práctica docente referida al desarrollo de páginas web, desarrollo de software educativo, aprender a utilizar las TIC en aula con entornos de aprendizaje virtual, cursos semipresenciales, entre otros, se evidencia que se encuentra en un nivel bueno, pues los docentes consideran necesario poderlos aplicar en el aula. En los lugares de mayor consulta, los docentes utilizan la casa y la universidad como lugares idóneos para el desarrollo del trabajo; conocen el manejo de plataformas de administración de cursos virtuales; cuentan con computadores y conexión a Internet y utilizan el video beam para proyectar presentaciones como recurso de clase en un buen nivel.

Matriz de resultados estudiantes. Las preguntas del estudiante están divididas en tres grupos según la batería de preguntas. De acuerdo con las figuras 9, 10 y 11 , se evidencia que los estudiantes cuentan con computador y conexión a Internet. Se percibe que hay una utilización de nivel muy bajo del computador. Los estudiantes utilizan en un nivel bueno otros equipos móviles como PDA, IPOD, Mp3, etc. Para el desarrollo de sus actividades y conocen en un nivel alto más de medios y tecnología que los docentes.

Los estudiantes utilizan recursos como procesadores de texto (Word), bases de datos (Access, dBase, Oracle, SQL...), paquetes de presentación (Power Point) en un buen nivel. Con respecto a 

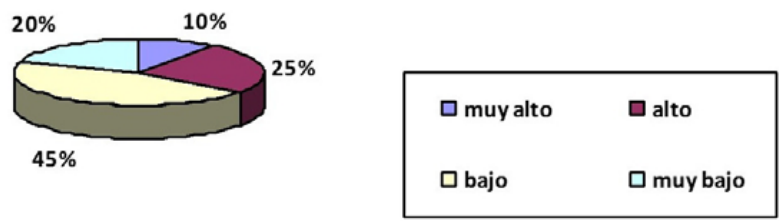

Figura. Componente técnico estudiantes. (TIC) grado de satisfación con recursos proporcionados por las IE
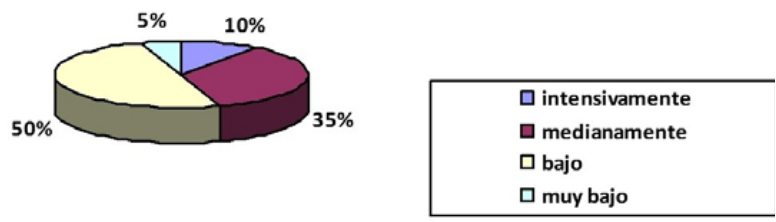

Figura 10. Componente técnico estudiantes. Utilización de recursos TIC

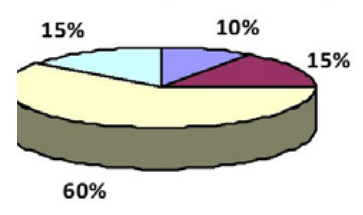

$\square$ muy alta $\square$ alta $\square$ media $\square$ baja

Fig. 11. Componente técnico estudiantes. Aceptación de plataforma y servicios

los paquetes estadísticos, muy pocos estudiantes utilizan este recurso. Con respecto a software de internet (Explorer, Netscape, Mozilla...), enciclopedias temáticas multimedia (Encarta, Adams... otros), simuladores y/o Software educativo en general, edición de páginas web (html, Publisher, frontpage...otros), hojas de cálculo (Excel), el nivel de los estudiantes es muy bueno.

Con respecto los equipos de que dispone la Institución para el desarrollo de tareas o actividades que requieran el uso sistemas de cómputo, las características de los equipos en cuanto a: velocidad de conexión y software instalado en general, son adecuados. Además, con respecto a los servicios en línea, los estudiantes perciben que son muy adecuados.

En cuanto a disponibilidad de múltiples tecnologías como recursos para la realización de las tareas académicas y conocimientos en informática básica e Internet, es muy importante. Los estudiantes consideran que los computadores y la tecnología deben estar integrados en el salón de clases es casi siempre importante.
Factor pedagógico. (Matriz de resultados directivos-estudiantes - docentes, según la batería de preguntas a factores pedagógico- comunicativo). La mayoría de portales institucionales destacan en su web site su Proyecto Educativo Institucional caracterizado como un proyecto flexible y permeable y de construcción permanente. Centra su acción formativa en competencias a la luz de las orientaciones emitidas por los órganos colegiados y las políticas públicas ministeriales. Se enmarca dentro de la concepción estratégica a partir de objetivos estratégicos, donde el eje central del proceso es el estudiante; por ello la institución debe velar por una excelencia en la enseñanza en todos los niveles. Se observa una estrecha coordinación con el epistemológico, pedagógico y político, como base para la formación de estudiantes críticos, creativos, sujeto del proceso de aprendizaje y capaces de transformar la realidad. El proyecto muestra una clara intencionalidad de corte profesionalizante, ya que incluye aspectos investigativos relevantes que orientan los procesos académicos hacia la consolidación de estructuras investigativas sustentadas académicamente.

De otro lado en algunos portales, se observa una estructura consolidada en lo referente al concepto de educación virtual. Este concepto está sustentado en una plataforma (EPIC) se destaca el "curso sello", el cual consiste en tres cursos virtuales orientados al conocimiento de la plataforma virtual, la metodología de aprendizaje y la forma como el estudiante puede navegar por los contenidos, que en esencia son de carácter instruccional (Figura 12).
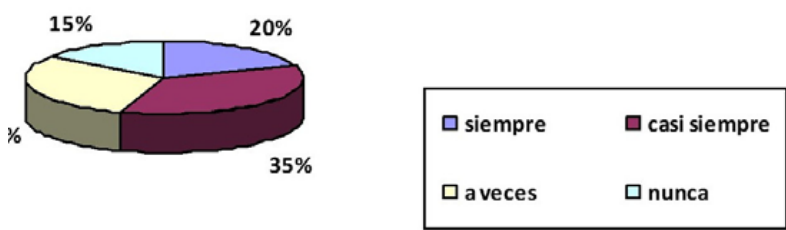

Figura 12. Componente comunicativo. Uso de recursos TIC en redes sociales académicas y otros

En varias web site no aparece explícitamente información sobre la cual se pueda realizar un análisis acerca de la estructura pedagógica. Se hace visible el modelo EPIC, lo que hace suponer la presencia de un modelo único soportado en tic, que permita el sostenimiento de los programas 
Estudio preliminar sobre el uso de tecnologías de información y comunicación en instituciones de educación superior de la Red Ilumno

académicos ofertados bajo la modalidad UDI GLOBAL LEARNING

Al realizar la observación de los resultados obtenidos luego de la aplicación de las encuestas, se obtuvo información relevante entorno al uso de TIC en procesos pedagógicos y en procesos comunicativos. En lo relacionada al factor pedagógico, se puede deducir que existe una clara influencia de las TIC en los procesos educativos. Para ello las instituciones ofrecen sistemas de formación en uso de plataformas computacionales, en particular aquellas instituciones que soportan sus procesos de educación a distancia y "virtual", en el LMS EPIC. Han dispuesto de programas de capacitación (curso sello, curso dirigido hacia la producción de contenidos, cursos de formación en tutoría a distancia y virtual). En ese sentido se enfatiza en factores de tipo comunicativo, que forman parte integral de estos procesos centrados en su mayoría en los estudiantes que cursan programas de distancia y virtuales.

De otro lado, se observa que la mayoría de los docentes poseen una formación básica en uso de TIC, que les permite en cierta medida desempeñarse en sus funciones como docentes y responder de alguna manera a la estructura de plataformas computacionales. Al respecto, las plataformas con mayor familiaridad en el uso son: Blackboard, Moodle, EPIC; la mayoría de ellas en la actualidad tiene cursos virtuales y estudiantes matriculados en programas académicos virtuales y a distancia.

Así mismo en el orden de usabilidad de las Tic, se encuentra que un amplio sector de docentes, estudiantes y directivos docentes tiene acceso a una gama mediana y alta de dispositivos móviles (DM), lo que de hecho facilita una comunicación permanente a través de redes sociales, además amplía las esferas de la escuela en el sentido de estar en condiciones de responder en periodos de tiempo más amplios que los registrados en los programas académicos. La mayoría manifiesta su interés por pertenecer a las redes sociales y establecer campos de comunicación alternativos a través de blogs, foros, y wiki; asimismo, un gran sector de profesores manifiesta pertenecer a redes académicas. Una parte de los docentes y directivos docen- tes manifiestan haber cursado y haber recibido formación a través de los DM (figura 13).
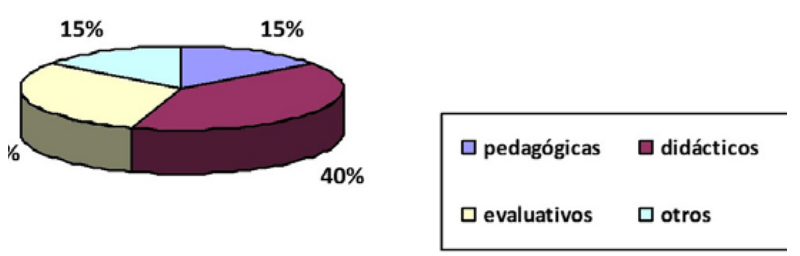

Figura 13. Componente comunicativo. Apropiación de recursos TIC en funciones académicas y otros

Se observa un gran interés por parte de los encuestados (directivos, docentes, estudiantes) en capacitarse y actualizarse en el uso de TIC, manifiesto en la receptividad a los cursos de formación ofrecidos por las diferentes IES.

\section{Discusión y Conclusiones}

En primera instancia, los investigadores plantearon la realización de un estudio comparativo sobre el uso y la apropiación de TIC en nueve de las IES de la red ILUMNO, con el cual iniciaron la ruta de investigación; pero, al avanzar en el proceso metodológico, se hallaron varios obstáculos de corte procedimental que hicieron cambiar las preguntas de investigación $y$, por consiguiente, algunas partes del diseño metodológico. Sin embargo, se conservaron las fases contempladas para su desarrollo, así como los factores (pedagógico, tecnológico, comunicativo, organizacional) que estructuraron el proceso investigativo y que se incluyen en este documento.

Con el Estudio preliminar sobre el uso de Tecnologías de Información y Comunicación TIC en Educación Virtual en Instituciones de Educación Superior que pertenecen a la red ILUMNO se logró alcanzar las metas propuestas que, en general, fueron las siguientes:

- Recopilar, sistematizar y analizar la información sobre el uso de las Tecnologías de Información y Comunicación TIC en Instituciones de Educación Superior que conforman la red ILUMNO, a través de la información presentada por cada una de las instituciones en los portales institucionales y los sitios web. 
En particular, se visitaron las páginas correspondientes a la zona informativa institucional y al sistema de educación a distancia y virtual.

- Contrastar y sistematizar la información obtenida con los datos presentados a través de los sitios web institucionales.

- Estructurar la batería de instrumentos diseñados para este estudio y validados por expertos externos.

- Establecer contactos con directores de los programas de investigación y hacerlos partícipes del estudio.

- Plantear alrededor de este estudio la importancia que tiene el hecho de conformar redes de aprendizaje en forma de redes sociales que integren a este nivel las nueve instituciones de educación superior que conforman la red ILUMNO.

- Profundizar en aspectos teóricos sobre el uso de TIC en procesos educativos y en particular en lo referente a procesos educativos virtuales.

- Consolidar la información obtenida, analizarla y determinar las divergencias y convergencias que presenta cada uno de las instituciones que conforman el estudio.

- Analizar los resultados obtenidos a partir de la aplicación de los instrumentos diseñados en los factores pedagógico, comunicativo, organizacional, y tecnológico.

- Participar en eventos nacionales para la difusión de los avances y resultados parciales del estudio.

Factores como el pedagógico, comunicativo, organizacional, tecnológico forman parte de los estudios y pueden ser abordados desde la comprensión de la realidad educativa del país y de América Latina y el Caribe, puesto que esta dinámica de las TIC se ha convertido en una megatendencia, que aún no ha sido explicada desde estos cuatro factores. Por ello se hace necesario que este estudio continúe, retomando estos factores e incorporando el factor de investigación. Por lo tanto se recomienda:

- Generar mayores espacios para la formulación, presentación y desarrollo de propuestas investigativas que generen respuestas a las problemáticas y calculen los impactos que las TIC han producido en el sector educativo y, en particular, en la IES que conforman la red ILUMNO.

- Construir un espacio de comunicación entre los grupos de investigación y los investigadores en el campo de la educación en cada una de las IES de la red ILUMNO para conformar una masa crítica que contribuya al fortalecimiento de una cultura investigativa del sector, propiciando la publicación de avances y resultados investigativos que se desarrollan al interior de las IES.

- Formular unas políticas institucionales a nivel de investigación orientada al establecimiento de unos planes, programas y proyectos, como también unas líneas de investigación transversal, orientada desde la Red ILUMNO.

- Fortalecer el espacio para la socialización de proyectos y programas de investigación que presenta la Red ILUMNO a través del seminario virtual.

- Convocar a la comunidad de investigadores de la red ILUMNO a un Primer Encuentro de Investigadores en Educación de la IES que conforman la Red ILUMNO (presencial/ virtual), con el fin de socializar investigaciones y resultados y consolidar la red de investigadores de la Red ILUMNO.

\section{RefERENCIAS BibliográficAs}

Aguilar, G.T. (2011). Ontología Cyborg. El cuerpo en la nueva sociedad tecnológica. Barcelona: Gedisa.

Aprender y Enseñar en la Cultura Digital. (2011). VII Foro latinoamericano de Educación experiencias y aplicaciones en el aula. Buenos Aires: Fundación Santillana.

Banco Mundial - OCDE. (2012). Serie Evaluación de Políticas Nacionales de Educación. Bogotá.

Bateson, G. (1984). Espíritu y naturaleza: una unidad necesaria (avances en teoría de sistemas, complejidad y ciencias humanas). Nueva York: Bantam Books.

Deleuze, G. y Guattari, F. (1990). Rizoma (introducción). Valencia: Pre-Textos.

Dussel I. y Quevedo L. (2010) Los desafíos Pedagógicos ante el mundo digital. Buenos Aires: Fundación Santillana.

Fundación Telefónica. (2014). Las TIC en la Educación Digital del tercer Milenio. III Foro Internacional de Valparaíso. 
Estudio preliminar sobre el uso de tecnologías de información y comunicación en instituciones de educación superior de la Red Ilumno

Valparaíso: Ariel.

Lévy. P. (1999). ¿Qué es lo virtual? Buenos Aires: Paidós.

McLuhan, M. (1967). El medio es el mensaje. Buenos Aires: Paidós.

Méndez, C. (2011). Metodología: Guía para elaborar diseños.

Rama, C. y Morocho, M. La Educación a Distancia y Virtual en Ecuador, una nueva realidad universitaria. Quito: Editorial UTPL- Virtual Educa.

Serres M. (1994). Historia de las Ciencias. Madrid: Editorial Cátedra.

Carta Universitaria Rio. (2014). Tercer encuentro Internacional de Rectores Universia-Rio de Janeiro.

Vaillant, D. (2013). Integración TIC en sistemas de formación Inicial y continua para la educación básica en América Latina. Buenos Aires: UNICEF.

Virilio, P. (2011). El arte del motor- Aceleración y realidad virtual (3a ed.). Buenos Aires: Editorial Manantial. 